SPECIAL ARTICLE

\title{
Leprosy immunology-some aspects of the role of the immune system in the pathogenesis of disease
}

T GODAL

Laboratory for Immunology, Norwegian Radium Hospital, Montebello, Oslo 3, Norway

We are grateful to Dr Godal for permission to present this adaptation of his slide and videotape on leprosy immunology at one of the teaching sessions of the XII International Leprosy Congress held in New Delhi, February 1984. The figures and diagrams have been taken from transparencies and we apologise for some loss of definition. Editor.

The immunology of leprosy has been a subject of extensive research for the last 15 years. Important progress has been made in a number of areas, and support for the overall concept shown in Figure 1 is steadily being accumulated. In Figure 1, time after exposure to Mycobacterium leprae is shown along the horizontal axis and strength of cell-mediated immunity along the vertical axis.

The concept proposes that among those who become exposed to $M$. leprae, the great majority appear to develop an effective immune response sufficiently rapidly to arrest $M$. leprae infection before overt clinicaldisease is precipitated. This I will call subclinicalinfection. Only in a minority of subjects does the disease apparently become clinically expressed. Towards the tuberculoid end, considerable evidence suggests that the immune response to $M$. leprae is the major cause of lesions, while towards the lepromatous end of the spectrum accumulation of vast numbers of bacilli in infiltrating host cells plays an important role.

The precise detection of subclinical infection is of fundamental importance to a more complete epidemiological understanding of leprosy. This has not yet been achieved. However, significant advances have been made recently in this area by development of $M$. leprae-specific serological techniques as pioneered by Abe. More recently the employment of a chemically defined and unique antigen of $M$. leprae, namely the phenoicglycolipid identified by Brennan and his co-workers, appears promising. In this and related areas the development of monoclonal antibodies is rapidly becoming important to leprosy immunology. The difference between conventional and monoclonal antibodies is illustrated in Figure 2. As you see in Figure 2, if an animal is immunized with an antigen, the antiserum will contain antibodies to many different structures on the antigen. However, each lymphocyte in the animal produces only one type of antibody. This is utilized in the production of monoclonal antibodies. This is illustrated on the right side of Figure 2. In the production of monoclonal antibodies each lymphocyte is fused with a myeloma cell and cloned. In this way antibodies of identical specificities are produced. Moreover, they can be produced in unlimited amounts, because the myeloma cell has conferred immortality on the lymphocyte. 


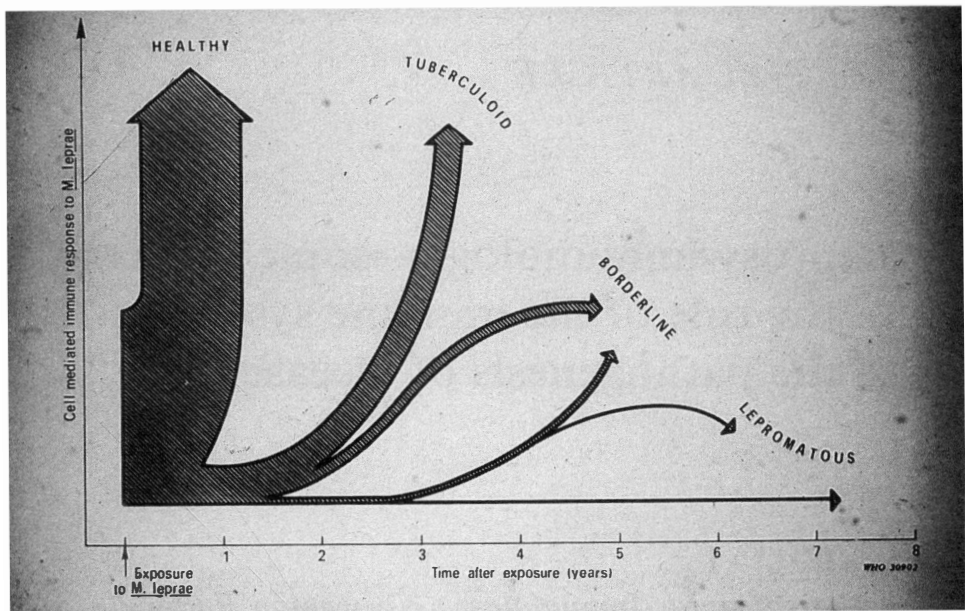

Figure 1. From Godal et al. Bull L'Inst Pasteur, 1979; 72: 273.

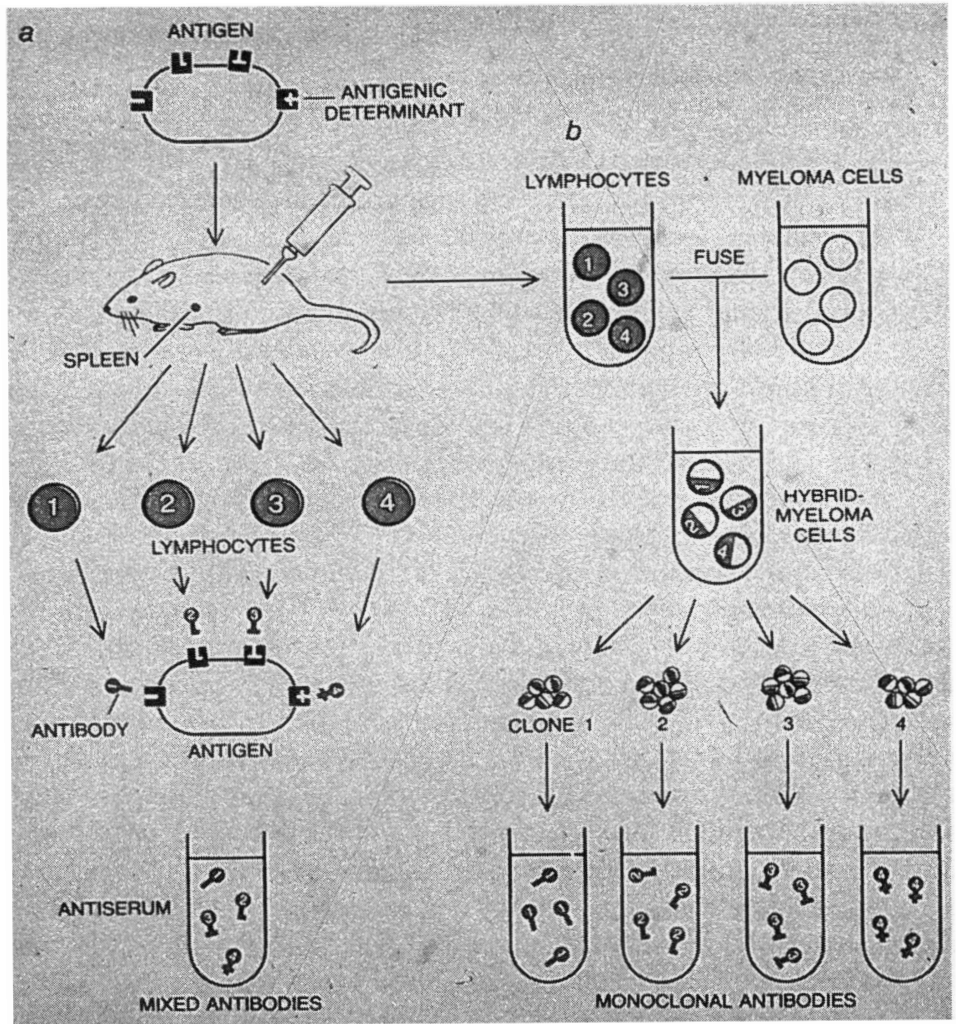

Figure 2. From Milstein, C., Sci Amer, 1980; 243: 56. 


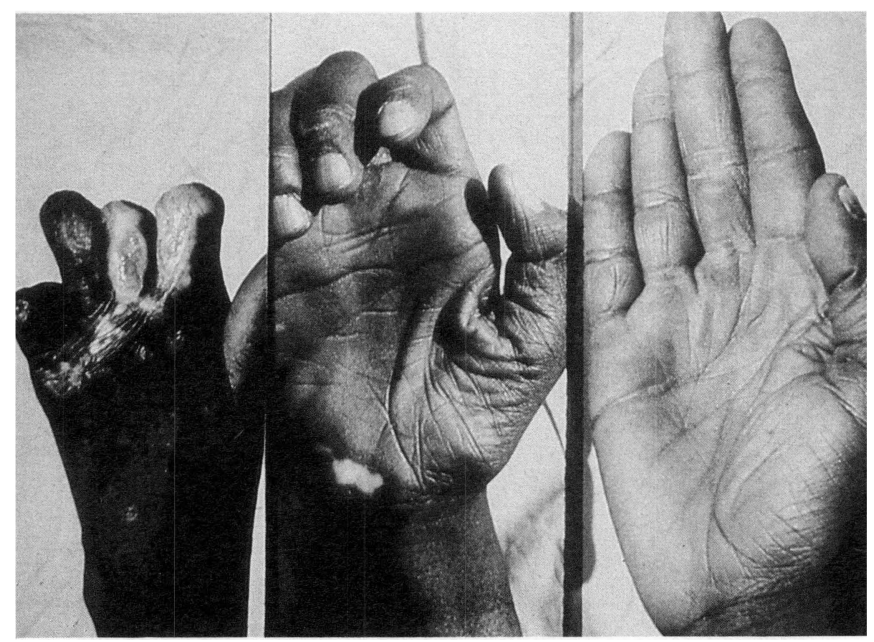

Figure 3

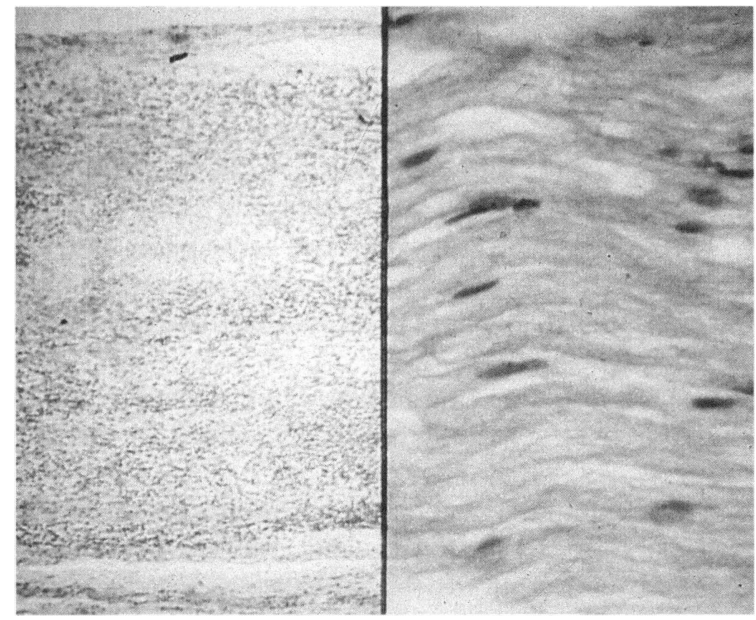

Figure 4

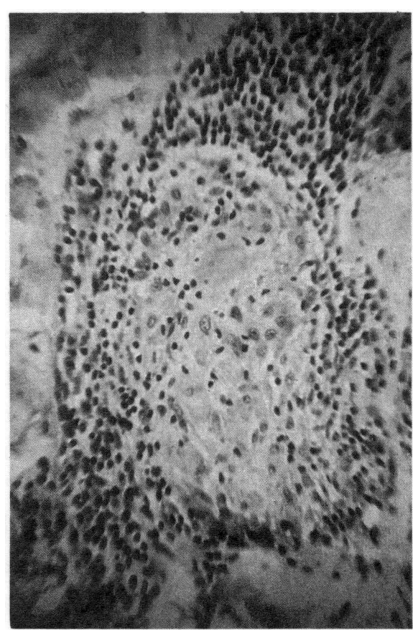

Figure 5

Two aspects of leprosy immunology are focused upon, namely nerve damage in borderline and tuberculoid patients, and the nature of the immunological deficiency in lepromatous leprosy.

Nerve damage in leprosy is of key importance, since this, as illustrated in Figure 3, is a major cause of deformity. Deformity often results from loss of sensation and loss of motor nerve function. If one looks histopathologically at damaged nerves in borderline and tuberculoid patients, as shown in Figure 4, the regular cable-like structure may be completely broken down by infiltrating inflammatory cells. Actually, as shown in Figure 5, there is granuloma formation within the nerves with lymphocytes, macrophages and epithelioid cells. A considerable body of evidence suggests that this granuloma formation within the nerves results from immunological attack from the host on leprosy bacilli hiding within the nerves. Thus, whenever recognized by the host immune system, $\mathrm{T}$ lymphocytes will become attracted to these sites and release various factors called lymphokines, 


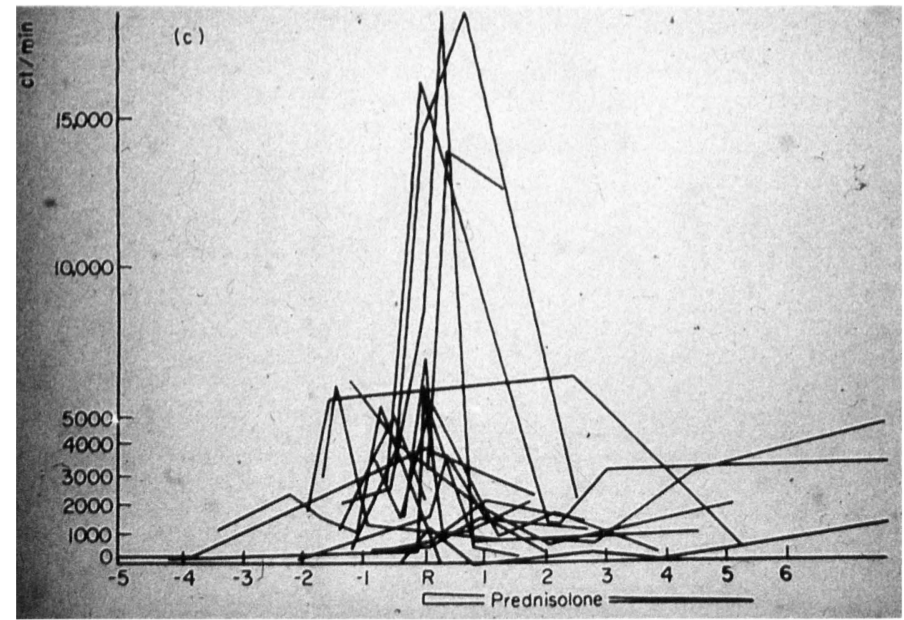

Figure 6. From Bjune et al. Clin exp Imm, 1976; 25, 85.

which in turn will attract and activate monocytes to kill bacteria that they will engulf. However, this attack will, as an unfortunate side-effect, also distort and damage nerve fibres and function. It is important from a clinical point of view that this type of nerve damage in leprosy may occur very rapidly. This is especially seen in reversal reactions, where there may be a rapid build up of immunological attack on leprosy bacilli. This is shown in Figure 6 taken from a prospective study carried out by Barnetson, Bjune and co-workers. Along the horizontal axis you see time in months before and after the development of reaction and along the vertical axis lymphocyte proliferation to $M$. leprae as measured by radiolabelled thymidine incorporation. The close association between reaction and lymphocyte proliferation is indeed very striking. It is therefore very important to treat such patients adequately as soon as possible, that is, they really have to be considered as emergency cases, otherwise nerve function may be permanently lost.

Let us now turn to lepromatous leprosy. The central question here is, what is going wrong in lepromatous leprosy? Why does the host system fail to attack the leprosy bacilli, which are thriving in the tissues in vast numbers?

It is well known from earlier studies that this immunological defect is remarkably specific to leprosy bacilli. This is illustrated in Figure 7. Here you see 3 treated patients and their lymphocyte proliferative response to $M$. leprae, BCG and PPD. As you can see, the patients responded strongly to BCG and PPD, but were completely negative to $M$. leprae. Thus, the defect is what we

\begin{tabular}{|c|rrr|}
\hline \multirow{2}{*}{$\begin{array}{c}\text { Patient } \\
\text { No. }\end{array}$} & \multicolumn{3}{|c|}{ Antigens } \\
\cline { 2 - 4 } & BCG & PPD & M. leprae \\
\hline 322 & 6.6 & 12.4 & 0.3 \\
327 & 35.7 & 82.4 & 0.6 \\
328 & 16.2 & 66.3 & 0.7 \\
\hline Mean & 19.5 & 53.7 & 0.5 \\
\hline
\end{tabular}

Figure 7. Lymphocyte transformation (uptake of ${ }^{3} \mathrm{H}$ thymidine, $\mathrm{T} / \mathrm{C}$ ratio) in leukocyte cultures of treated lepromatous patients. (From Godal et al. Scand J Immunol, 1972; 1: 311. 
immunologists call antigen specific. Since it is well known from a large number of studies, including studies on T-cell-deficient animals, that it is the T-cell that has the capacity to mediate specific immunity to intracellular bacilli such as the leprosy bacillus, one has for a long time suspected that $\mathrm{T}$-cells play a central role in the defect of lepromatous leprosy. The mechanisms involved in T-cell activation and T-cell-mediated intracellular killing of mycobacteria have advanced considerably during recent years and allow a more detailed analysis of the defect in lepromatous leprosy. Thus, we will here now first consider the basic concepts of T-cell activation and then discuss recent findings, which suggest more precisely the nature of the defect in lepromatous leprosy.

The T-cell response may be subdivided into three parts (Figure 8), the afferent limb or inductive

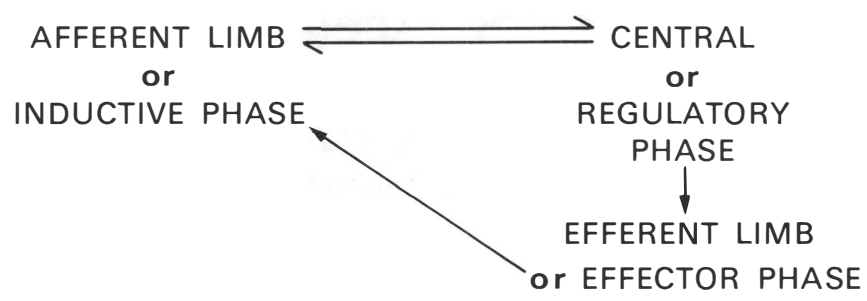

Figure 8. T-cell response to $M$. leprae.

phase, the central or regulatory phase or level, and the eff erent limb or eff ector phase. With regard to the afferent limb, we have known for a number of years that $\mathrm{T}$-cells do not see antigen alone, but that antigen is presented to the T-cell by other cells, so-called antigen-presenting cells, which include monocytes, macrophages or dendritic cells. The Langerhans cells of the skin also belong to this cell category.

How antigen-presenting cells interact with T-cells is not yet a fully understood process. It appears they actually talk to each other, that is to say, it is a mutually dependent, highly sophisticated process (Figure 9). The antigen-presenting cells have on their surface antigen derived from, in our case, $M$. leprae and high concentrations of HLA-DR molecules, both of which are required for T-cell activation. In addition there is evidence that the antigen-presenting cell produces a factor, interleukin 1 (IL-1), which is required for T-cell activation. However, the production of IL-1, as well as the level of HLA-DR expression, may actually be under T-cell control, as illustrated to the left by the $T_{1}$ cell. The activation of T-cells leads to two clearly distinguishable phenomena: 1 , one subset of T-cells, here called $\mathrm{T}_{2}$, starts to produce a factor required for T-cell proliferation and production of lymphokines. This factor is called interleukin 2 (IL-2); and 2, some T-cells, here called $\mathrm{T}_{3}$, will develop receptors for IL-2 and thereby become able to respond to IL-2.

Although I have for simplicity depicted three T-cell functions as located to three subsets of $\mathrm{T}$-cells $\left(\mathrm{T}_{1}-\mathrm{T}_{3}\right)$, they may actually be largely overlapping populations.

This part of the immune response, the afferent limb, sets the stage for T-cell proliferation and interleukin production, which may be called the central level of the immune response. The central level may also be called the regulatory level, because T-cells are controlled by other T-cells, so-called suppressor cells, and this regulation is often called the suppressor circuit (or the suppressor circus! because there are many unclear aspects and controversial issues about the suppressor circuit) (Figure 10). These suppressor cells may have the T4 or the T8 phenotype and are thus not limited to T8 cells. Suppressor cells may interfere with T-cell activation in various ways, for example by blocking induction of IL-2 receptors or by blocking IL-2 production.

Let us now consider the third part of the T-cell response, the so-called efferent limb (Figure 11). How do T-cells effect their attack on $M$. leprae and related organisms? It appears that T-cells mainly 


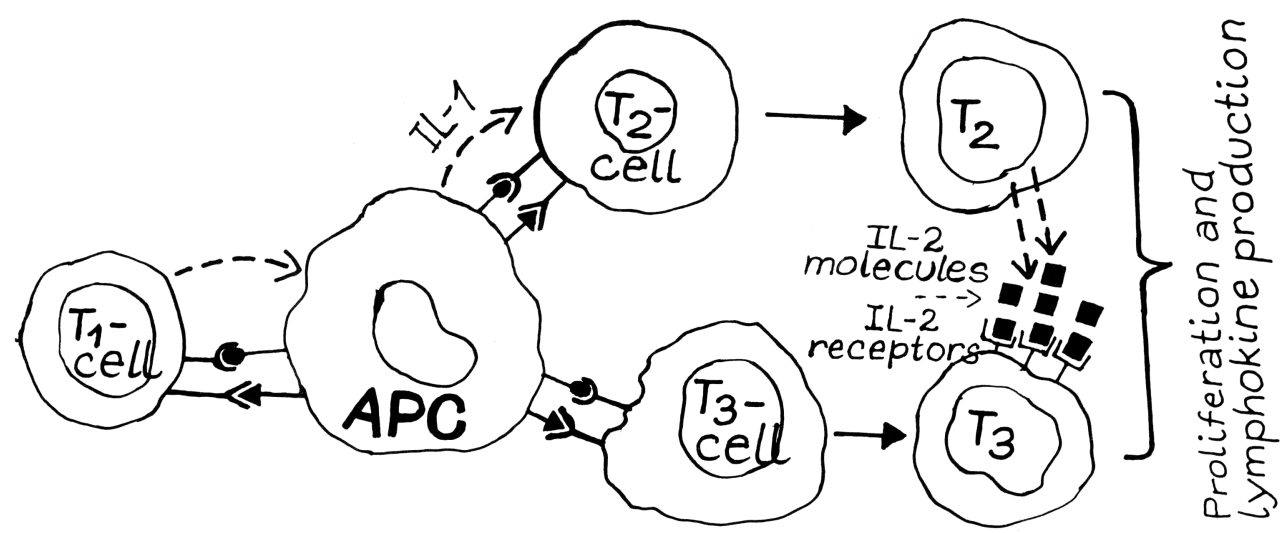

Figure 9. Afferent limb. ๑, HLA-DR molecule ('Ia'); $\mathbf{\Delta}$, antigen; APC, antigen presenting cell (monocytes, macrophages, dendritic cells, Langerhans cells, B-cells etc.) IL-1, Interleukin 1; IL-2 Interleukin 2 (or T-cell growth factor).

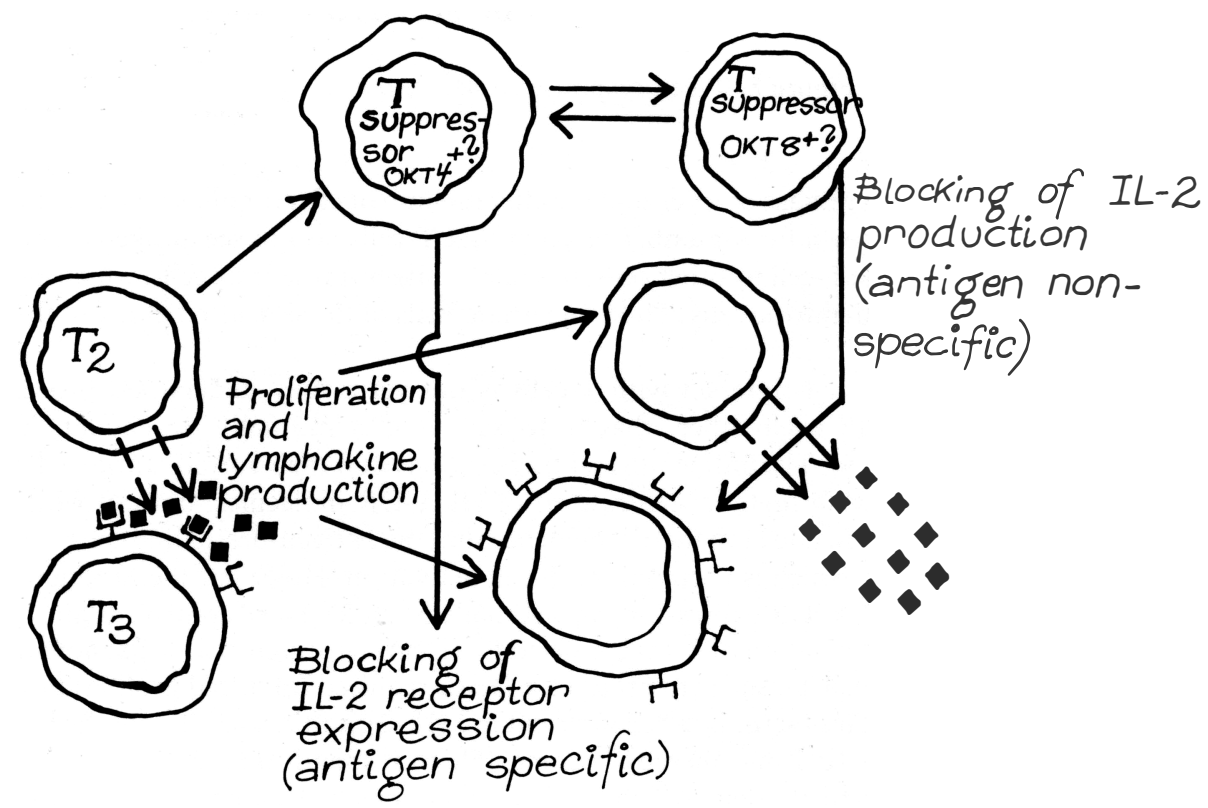

Figure 10. Central or regulatory level with suppressor circuit.

orchestrate or conduct the attack by production of lymphokines, some of which have chemotactic properties and attract monocytes from the blood into the sites where $M$. leprae has been detected; and other lymphokines, one called macrophage activation factor (MAF), probably identical with $\gamma$-interferon, activate the macrophage to kill and digest the bacteria they have internalized.

We may now return to the question of what is going wrong in lepromatous leprosy. It would be apparent that there are many places where things could go wrong: 1, the antigen-presenting cells may be compromised; 2 , T-cells may lack receptors for $M$. leprae antigens; 3 , patients may have developed an overwhelming suppressor circuit that could suppress IL-2 receptor induction or IL-2 production; and 4 , there could be a defect in the efferent limb. 


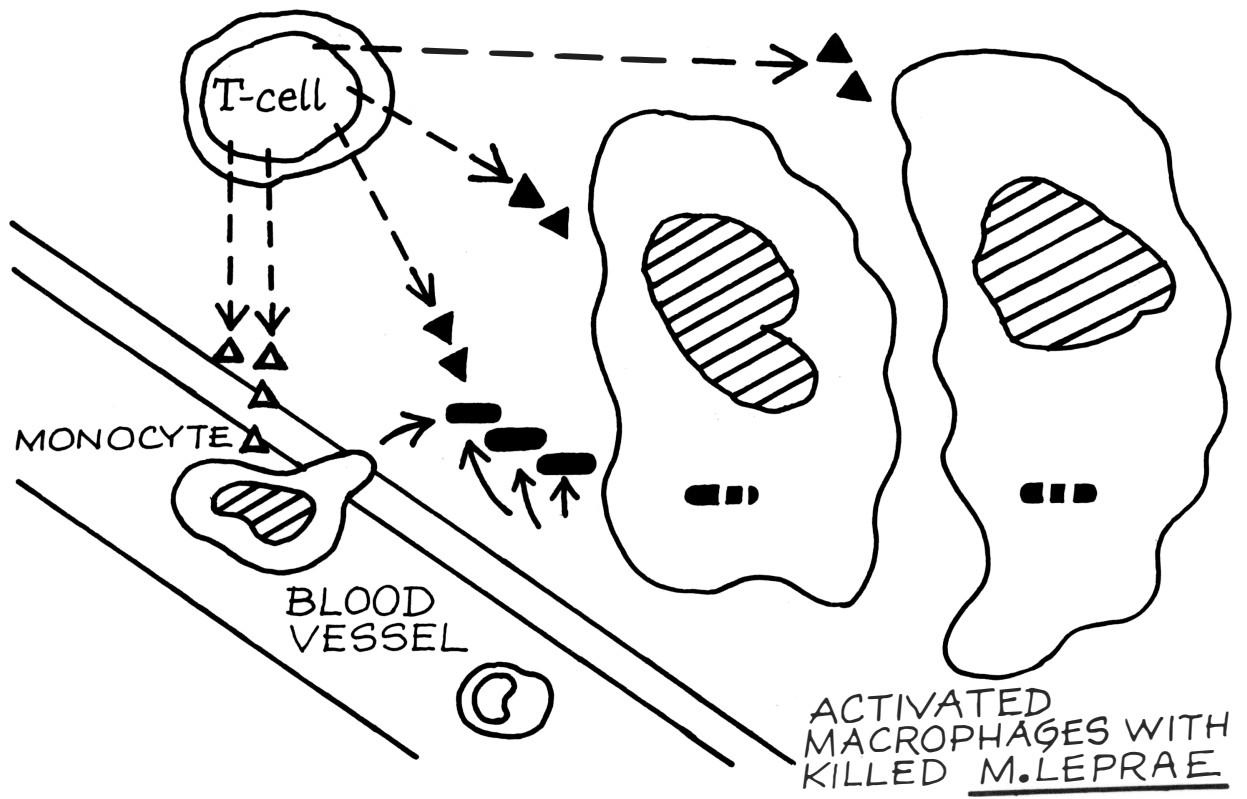

Figure 11. Efferent limb. $\longrightarrow$, live M. leprae; $\Delta$, chemotactic factors; $\Delta$, macrophage activation factor $\left(\mathrm{T}_{\gamma}\right.$-Interferon?).

Space does not allow us to consider in detail all the experimental data which may be considered for or against any of these possibilities. However, data have steadily accumulated in recent years that provide further evidence that the def ect is located at the central or regulatory level (Figure 12). Several investigators, especially Mehra and Bloom, have detected suppressor cells in lepromatous leprosy. Finally, Haregewoin in Addis Ababa in collaboration with Salim Mustafa and myself has shown that lepromatous T-cells fail to produce IL-2, but if given IL-2 from external sources to lepromatous T-cells, the T-cells will now mount a proliferative response to $M$. leprae.

Combined these findings suggest that suppression of IL-2 production may be of central importance. A proposal for the nature of the def ect is outlined in Figures 10 and 13. Figure 10 shows the normal regulatory compartment and Figure 13 the aberrancies in lepromatous leprosy. These findings are encouraging because they suggest that these studies on the immunological nature of defect in lepromatous leprosy may lead to new approaches for restoring immunological competence in such patients. Hopefully some day termination of chemotherapy and prevention of drug resistance may become feasible in such patients.

(1) Defect in afferent, regulatory or effector compartment. Present evidence suggests that the defect is localized to the regulatory compartment. The two main reasons are:

(a) Increased suppressor cell activity (OKT8 ${ }^{+}$, antigen non-specific suppressor cells) has been observed in LL (Mehra \& Bloom).

(b) Proliferative T-cell response to $M$. leprae can be restored in vitro with IL-2 (Haregewoin et al.). Thus, the afferent limb appears intact and suppression of IL-2 production appears to be of key importance.

Figure 12. What is going wrong in lepromatous leprosy? 


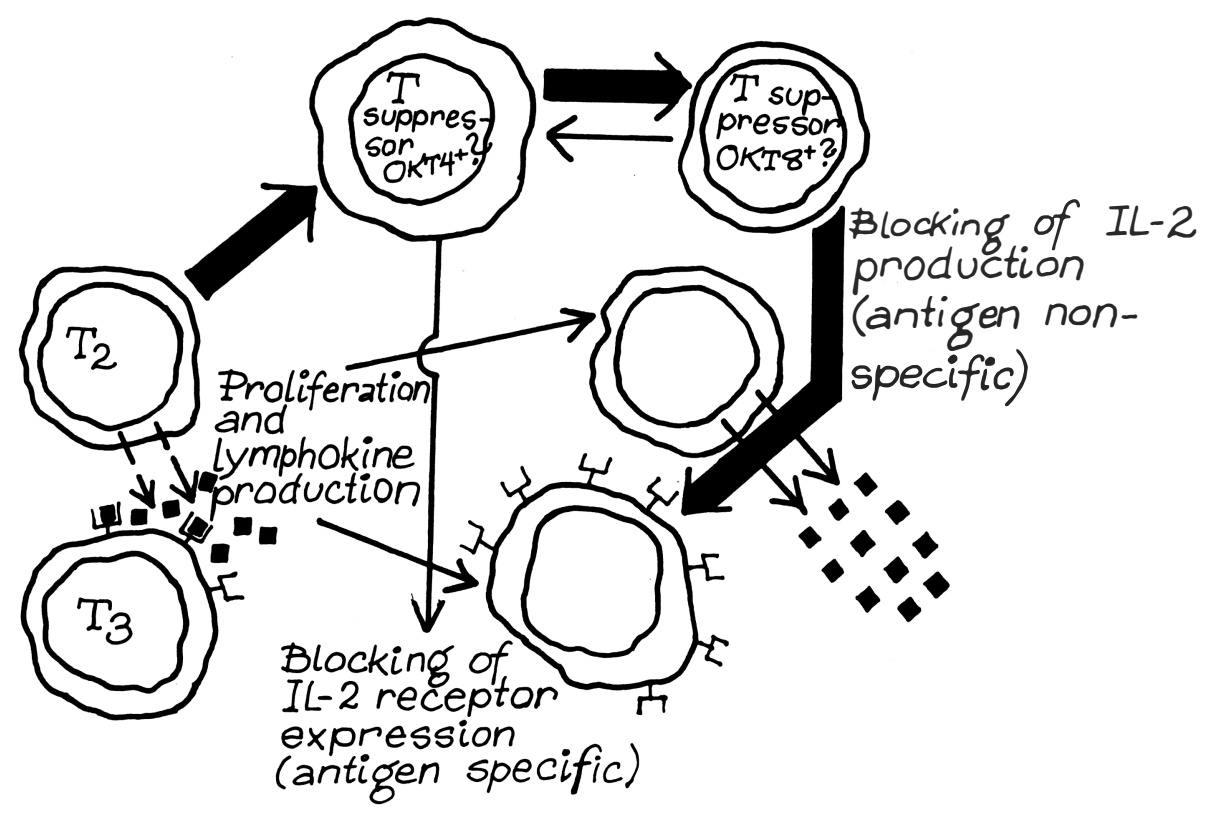

Figure 13. Defect in lepromatous leprosy.

In conclusion, the immune system is of central importance to the pathogenesis of various disease manifestations in leprosy. The main contribution of leprosy immunology so far has been at the conceptual level. But as you may well have noted during the XII International Congress of Leprosy, the stage is now set in a number of areas for exploring more direct contributions to leprosy control. 